\title{
LABORATORY AND FIELD RESULTS OF THE USE OF SPECTRAL INDUCED-POLARIZATION (SIP) METHOD FOR DETECTING ORGANIC AND INORGANIC CONTAMINANTS
}

\author{
Heikki Vanhala \\ Geological Survey of Finland (GTK) \\ P.O. BOX 96, FIN-02151 Espoo, Finland
}

\section{INTRODUCTION}

Environmental applications of the induced polarization (IP) and the spectral induced polarization (spectral IP or SIP) methods, i.e., the possibility to detect organic and inorganic contaminants in glacial soils, has been studied the Geological Survey of Finland (GTK) for some years. This presentation deals with the IP of natural and contaminated soils (the membrane polarization), and gives two field examples; an area with an organic (hydrocarbon) contamination (Fig. 1), and an area with an inorganic (heavy metal) contamination.

\section{THE SPECTRAL INDUCED POLARIZATION METHOD}

The IP method and its broadband extension, the spectral-IP method, were originally developed for ore exploration; the former largely at the 1950's, the later at the 1970's. In addition to exploration, IP has been used in groundwater and oil prospecting.

The idea of using the IP also with environmental problems - such as detecting hydrocarbons and organic solvents in soils - was introduced in the middle of 1980's by Olhoeft (1985). Sadowski (1988) measured phase and amplitude spectra of clean clay samples and organiccontaminated clay samples and observed a pronounced phase peak due to toluene contamination (the same kind of spectrum had been reported already by Olhoeft). Vanhala, Soininen, and Kukkonen (1992) described some effects caused by toluene, heptane, and ethylene glycol in till. Börner, Gruhne, and Schön (1993) measured clean and organic-contaminated clay and shaly sandstone samples. Vanhala (1996) discussed the effect of electrolyte conductivity on the phase spectrum and the possibility to use IP for detecting inorganic contaminations, and presented (Vanhala, 1997) laboratory and field results of the behaviour of phase spectrum of clean and oil contaminated sand and till.

The IP method is based on the concept that the resistivity of earth materials is a complex, frequency-dependent quantity

$\rho(\omega)=\rho^{\prime}(\omega)+i \rho^{\prime \prime}(\omega), i^{2}=-1$.

The IP is applied in the linear regime with the current density being sufficiently low, in which 
case the constitutive relation between the electric field $\mathbf{E}$ and the current density $\mathbf{J}$ is expressed by Ohm's law

$$
\mathbf{E}=\left[\rho^{\prime}(\omega)+i \rho^{\prime \prime}(\omega)\right] \mathbf{J}
$$

In the spectral IP, the amplitude and the phase shift are measured over a broad frequency range from about $0.01 \mathrm{~Hz}$ up to $10000 \mathrm{~Hz}$ and the resistivity is commonly in the form

$\rho(\omega)=|\rho(\omega)| \exp [i \phi(\omega)]$

where

$|\rho|=\left[\left(\rho^{\prime}\right)^{2}+\left(\rho^{\prime \prime}\right)^{3}\right]^{1 / 2}$

and

$\phi=\arctan \left(\rho^{\%} / \rho^{\prime \prime}\right)$

$|\rho|$ is the amplitude and $\phi$ the phase angle of the complex resistivity.

\section{PHASE SPECTRA OF CLEAN AND CONTAMINATED SOILS}

The phase spectrum of a glacial sediment is strongly dependent on its grain-size distribution: the strongest low-frequency (e.g., $1 \mathrm{~Hz}$ ) phase shifts (i.e., up to $30 \mathrm{mrad}$ ) are measured in silts and fine to medium-grained sands, while coarse sands and gravels are practically unpolarizable (i.e., $\left.\phi_{1 \mathrm{~Hz}}<1 \mathrm{mrad}\right)$ at low frequencies. The glacial clays also show weak low-frequency IP effects. Besides the grain size, the phase shift depends on the conductivity of the electrolyte: an increase in the conductivity of the electrolyte decreases the IP effect. The effect of moisture content is weak in fine and medium-grained sediments, but stronger in coarse sands and gravels, where the phase shift decreases with decreasing water content. A common feature of the spectra of glacial sediments is the increase in phase shift with increasing frequency, as seen in Fig. 2a.

The organic contaminants tested in the laboratory (waste oils, toluene, etc.) changed the phase spectrum and the resistivity of till and sand. The magnitude of the change of phase shift in artificially contaminated samples was typically within $+/-10 \mathrm{mrad}$ at low to medium frequencies. A typical feature observed in the laboratory was the change of the shape of the phase spectrum with increasing maturation time (of the contaminant). The organic contaminants had only small effect on the resistivity: often resistivity slightly decreased due to contamination. An example describing the effect of a waste oil on the phase spectrum and resistivity $(1 \mathrm{~Hz})$ on a till is given in Fig. $2 b$.

The phase shifts measured in soils and the contamination-generated changes are small, but still measurable under field conditions, and it seems that an organic contamination in homogeneous sand and till areas can be detected. In a larger group of soil types the spectra of contaminated sand and till are, however, not very abnormal so that under complex conditions (in the presence of clay layers, metallic objects, etc.) direct detection of contamination is uncertain. Under such conditions the instability of the phase spectrum of contaminated soil may, however, be diagnostic of contamination. 

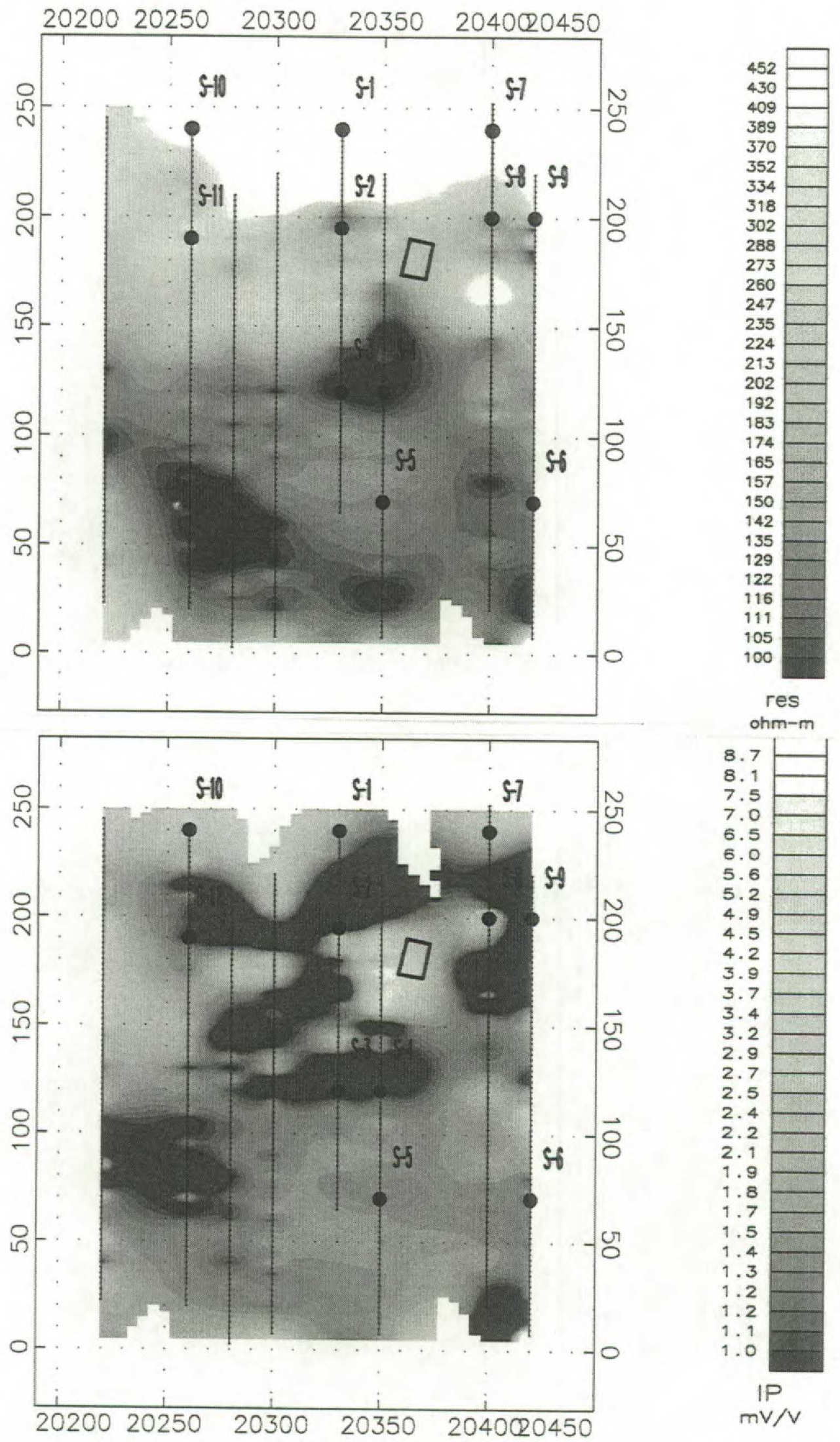

Fig. 1. The Röykkä test site. Apparent resistivity (upper) and chargeability (lower). The solid circles (S-1 - S-11) marks the sampling sites, and the open box (20360/170) the source of the contamination. 
(a)

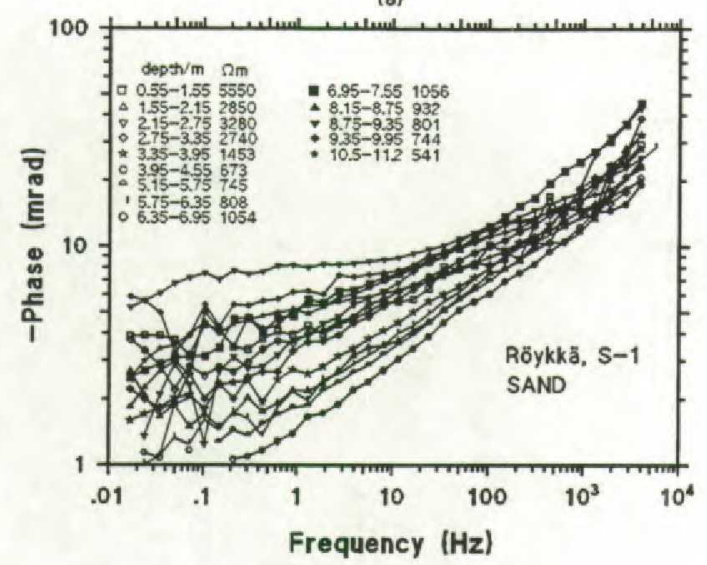

(b)

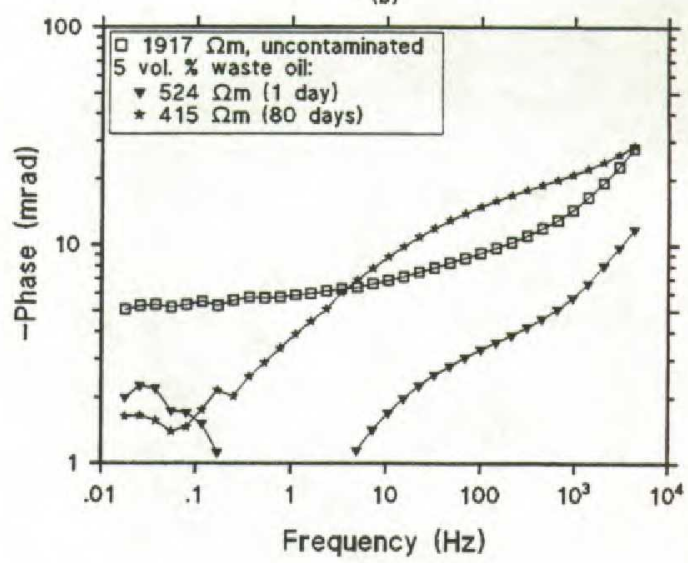

Fig. 2. (a) Laboratory phase spectra of samples from the sampling site S-1 (see Fig. 1). Soil is uncontaminated fine and medium-grained sand; water table at 10 metres; (b) Effect of waste oil on the phase spectrum and resistivity of till (Vanhala, 1997).

\section{FIELD EXAMPLES}

The first case is an area where a hydrocarbon contamination is situated close to groundwater area (Fig. 1, the aquifer is situated at the topside of the maps). Soil sampling for laboratory SIP measurements and organic analysis were carried out (see Fig 1.). A field geophysics includes EM-31 and IP ( $\mathrm{a}=5 \mathrm{~m}, \mathrm{n}=1-7)$ mapping and SIP measurements.

The second case is an area having a heavy-metal contamination due to wood preservatives used at a s

awmill. Sampling, laboratory SIP measurements, chemical analysis and field IP and SIP measurements were conducted.

\section{REFERENCES}

Börner, F., Gruhne, M. and Schön, J. 1993. Contamination indications derived from electrical properties in the low frequency range. Geophysical Prospecting 41, 83-98.

Olhoeft, G.R. 1985. Low frequency electrical properties. Geophysics 50, 2492-2503.

Sadowski, R.M. 1988. Clay-organic interactions. M.Sc. thesis, Colorado School of Mines.

Vanhala, H., 1996. Monitoring the integrity of liner construction using induced polarization. Proceedings of SAGEEP '96, Keystone, Colorado, 1017-1026.

Vanhala, H., 1997. Mapping oil-contaminated sand and till with the spectral induced-polarization (SIP) method. Geophysical prospecting, 45, 303-326.

Vanhala, H., Soininen, H. and Kukkonen, I. 1992. Detecting organic chemical contaminants by spectral-induced polarization method in glacial till environment. Geophysics 57, 1014-1017. 